\title{
Empirical Mode Decomposition in the Reduced-Order Modeling of Aeroelastic Systems
}

\author{
Young S. Lee \\ University of Illinois at Urbana-Champaign, Urbana, IL $61801^{1}$ \\ Alexander F. Vakakis \\ National Technical University of Athens, Athens, Greece ${ }^{2,3}$ \\ D. Michael McFarland \\ University of Illinois at Urbana-Champaign, Urbana, IL $61801^{4}$ \\ Gaëtan Kerschen \\ University of Liege, Liege, Belgium ${ }^{5}$ \\ Lawrence A. Bergman \\ University of Illinois at Urbana-Champaign, Urbana, IL $61801^{6}$
}

A relationship between Intrinsic Mode Functions (IMFs), derived from the Empirical Mode Decomposition (EMD), and the slow-flow model of a nonlinear dynamical system has been exploited in the development of the Slow Flow Model Identification (SFMI) method for strongly nonlinear systems, in which the physical parameters of such systems are identified from experimental data. Both the slow flows and IMFs provide the means to expand a general multicomponent signal in terms of a series of simpler, dominant, monocomponent signals. The slow flows are obtained analytically, for example through application of the method of complexification and averaging $(\mathrm{CxA})$, which transforms the equations of motion into a set of approximate equations in amplitude and phase for each modeled frequency component. In contrast, the EMD characterizes a signal through the envelope and phase of its elemental components, the IMFs. Thus, between nonlinear transitions, the equations derived using the CxA method govern the amplitude and phase of the modeled IMFs. Application of SFMI has, until now, been limited to low-dimensional systems subjected to impulsive excitation. Herein, the method is extended to identification of a planar rigid airfoil

${ }^{1}$ Postdoctoral Research Associate, Department of Aerospace Engineering, 104 S. Wright St., yslee4@uiuc.edu

${ }^{2}$ Professor, School of Applied Mathematical and Physical Sciences, P.O. Box 64042, GR-157 10, Zografos, Greece, vakakis@central.ntua.gr

${ }^{3}$ Adjunct Professor, Department of Mechanical Science and Engineering and Department of Aerospace Engineering, University of Illinois at Urbana-Champaign, Urbana, IL 61801

${ }^{4}$ Research Associate Professor, Department of Aerospace Engineering, 104 S. Wright St., dmmcf@uiuc.edu; Senior Member, AIAA

${ }^{5}$ Assistant Professor, Department of Aerospace and Mechanical Engineering, 1, Chemin des Chevreuils, g.kerschen@ulg.ac.be

${ }^{6}$ Professor, Department of Aerospace Engineering, 104 S. Wright St., lbergman@uiuc.edu; Associate Fellow, AIAA 
supported on nonlinear springs, subjected first to quasisteady aerodynamic loading, and then to aerodynamic loads encountered in transonic flow. The SFMI method is shown to be efficacious in these applications. These IMFs show promise for use in constructing reduced-order models of such systems, capable of capturing important interactions, such as energy flow between substructures, with a minimum of degrees of freedom.

\section{Introduction}

In several earlier papers, the authors demonstrated a relationship between Intrinsic Mode Functions (IMFs), derived from the Empirical Mode Decomposition (EMD), and the slow-flow model of a strongly nonlinear dynamical system ${ }^{1,2,3}$. This naturally led to the development of the Slow Flow Model Identification (SFMI) method for strongly nonlinear systems, in which the physical parameters of such systems could be identified from experimental data. Both the slow flows and IMFs provide the means to expand a relatively general multicomponent signal in terms of a series of simpler, monocomponent signals related to the dominant frequency components. The slow flows are obtained analytically through, for example, application of the method of complexification and averaging $\left(\mathrm{CxA}^{4}\right)$, which transforms the equations of motion into a set of approximate equations in amplitude and phase for each modeled frequency component. In contrast, the EMD characterizes a signal through the envelope and phase of its elemental components, the IMFs. Thus, between nonlinear transitions, the equations derived using the CxA method govern the amplitude and phase of the modeled IMFs. Application of SFMI has, until now, been limited to low-dimensional systems subjected to impulsive excitation. Herein, the method will be extended to identification of a planar rigid airfoil supported on nonlinear springs, subjected, first, to quasisteady aerodynamic loading and, second, to aerodynamic loads encountered in transonic flow. The SFMI method will be shown to be efficacious in these applications.

\section{Problem Formulation}

Consider the 2-DOF rigid wing (a flat plate or NACA0004 airfoil) depicted in Fig. 1, where application of a nonlinear attachment on the right of the figure is already incorporated for future use. The parameters for this typical section were adapted from Isogai's Case A modes ${ }^{5,6}$ :

$$
a=-0.2, x_{\alpha}=0.9 \mathrm{ft}, r_{\alpha}^{2}=0.87 \mathrm{ft}^{2}, \omega_{\alpha}=100 \mathrm{rad} / \mathrm{s}=15.92 \mathrm{~Hz}
$$

where the chord $(c=2 b)$ is regarded as unity.

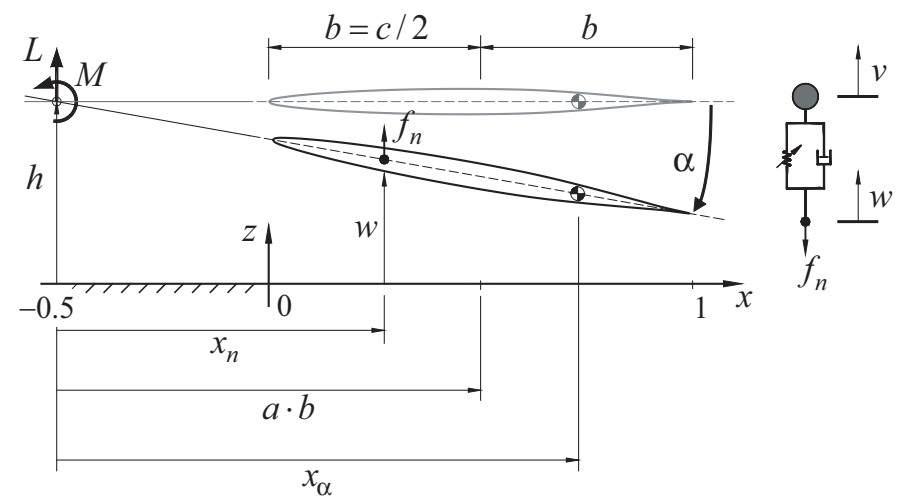

Fig. 1. Typical wing section for the transonic flutter model. 
The free (wind-off) vibration of this section is governed by

$$
\left[\begin{array}{cc}
1 & -x_{\alpha} \\
-x_{\alpha} & r_{\alpha}^{2}
\end{array}\right]\left(\begin{array}{c}
\ddot{h} \\
\ddot{\alpha}
\end{array}\right)+\left[\begin{array}{cc}
\omega_{h}^{2} & 0 \\
0 & r_{\alpha}^{2} \omega_{\alpha}^{2}
\end{array}\right]\left(\begin{array}{l}
h \\
\alpha
\end{array}\right)=\left(\begin{array}{l}
0 \\
0
\end{array}\right)
$$

Regarding $\omega_{h}$ as a structural parameter, and solving a generalized eigenvalue problem in the mass and stiffness matrices, we obtain the natural frequencies and modes of free vibration. The corresponding physical displacements of the wing are easily constructed from the eigenvectors, which will in general depend on the choice of $\omega_{h}$. Initial conditions for aeroelastic analysis are arbitrary, but it is convenient to assume zero initial displacements and an initial velocity in the shape of the first (lower-frequency) natural mode.

For example, if $\omega_{h}=\omega_{\alpha}$, then we obtain the information about the two structural modes; that is, the two natural frequencies are $\omega_{1}=71.34 \mathrm{rad} / \mathrm{s}=11.35 \mathrm{~Hz}$ for the out-of-phase mode with the eigenvector $u_{1}=(1,-1.072)^{T}$, and $\omega_{2}=533.77 \mathrm{rad} / \mathrm{s}=84.95 \mathrm{~Hz}$ for the in-phase mode with the eigenvector $u_{1}=$ $(1,1.072)^{T}$. The corresponding physical displacements of the wing can be constructed from these two eigenvectors ${ }^{5}$. The first mode (out-of-phase) with the frequency ratio $\omega_{1} / \omega_{\alpha}=0.713$ exhibits a pivot point at $-3.87 b$ from the elastic axis, whereas the pivot point for the second mode (in-phase) with $\omega_{2} / \omega_{\alpha}=5.340$ is at $-0.13 b$ from the elastic axis. Therefore, the first mode carries more of a bending mode, whereas the second mode, more torsional.

Two approaches were taken to the simulation of the system. In the first, a simple program was written in Fortran to calculate the heave and pitch responses, assuming simple, quasisteady aerodynamics. Nonlinear, cubic-hardening springs were added to the model along with linear, viscous dashpots, all acting at the elastic axis. In the second approach, to more realistically model the aerodynamic loads encountered in transonic flow, the CAPTSDv computer program developed by NASA was adapted. This code solves a finitedifference approximation to the transonic small-disturbance equations using an approximate-factorization algorithm ${ }^{7}$, and has been used in several investigations of limit cycle oscillation (see, e.g., ${ }^{8,9}$ ).

\section{Preliminary Results}

Two cases for the aeroelastic responses and the lift coefficient under the flow speed $U=310 \mathrm{ft} / \mathrm{s}$ (which belongs to a low-speed subsonic regime) will be considered; that is, responses with and without structural damping. The initial conditions for the simulations were given with zero initial displacements and an initial velocity in the shape of the first natural mode. In addition to being convenient, this choice corresponds to the appearance of transonic flutter (see, for example, ${ }^{5,6}$ ) in the first natural mode of the wing structure.

\section{A. Aeroelastic responses with no structural damping}

Figure 2 (a) depicts the heave and pitch mode responses with no damping in the wing structure for the low subsonic flow speed $U=310 \mathrm{ft} / \mathrm{s}$, and Fig. 2 (b), the close-up for $t \in[1,1.5]$. The responses in both modes apparently exhibit modulations of envelopes, which are almost in-phase. This modulation behavior is evident from the wavelet transforms in Fig. 2 (c). That is, when the initial conditions were given to excite the first natural mode at $\omega_{1}$, most of the modal energy is concentrated near $3 \omega_{1} \triangleq \Omega$. The aeroelastic modes result in various harmonics with energy distributed mainly at the integer factors of $\Omega$. All these harmonics represent quasiperiodic aeroelastic responses under the presence of no structural damping.

Furthermore, the WTs depict two predominant regimes: the mixed-mode response around $\omega_{2}$ and another beat response but with the component at $\Omega$ being prominently dominant. Performing the enhanced EMD analysis on these time series, these two dominant regimes are obviously separated as the first two intrinsic mode functions (IMFs) in Figs. 2 (d) and (e). The third IMF represents the component at $\omega_{1} / 2$. Note that the first IMFs, apparently containing several harmonics, cannot be separated further, and thus can be regarded as a single (weak) intrinsic mode oscillation (IMO). The IMO is defined as a linear oscillation but with narrow-bandwidth, time-varying frequency and damping. Instantaneous frequencies obtained from the first two IMFs correspond to the main frequency-energy distribution on the WTs, where the instantaneous frequency from the first IMF clearly captures the modulated behavior with several harmonic 
components. Figure 2 (f) depicts reconstruction of the original responses with the IMFs obtained, which should be obvious by virtue of decomposition.

The partial time series of the total lift coefficient (with $\omega_{h}=\omega_{\alpha} / 2$ ) is plotted in Fig. 3 (a), the whole of which is very close to an LCO driven and bounded entirely by nonlinear aerodynamic forces. The portion of the response that is strongly influenced by initial conditions is quite brief and cannot be discerned on this plot, but the aerodynamically dominated response includes a large "burst" peaking around $t=0.6 \mathrm{sec}$ into the time series, followed by a smaller-amplitude, unsteady oscillatory response.

Although the EMD method is frequently said to be a filtering tool, the instability of the EMD method hinders obtaining clean narrow-bandwidth IMFs from the time series of lift coefficient containing very high-frequency noise-like components. Since these high-frequency contents are not dominant, the time series were smoothed by applying a low-pass filter and interpolation (Fig. 3 (a)). Then, the three dominant IMFs of narrow bandwidth are obtained in Fig. 3 (b); the original signal can be reconstructed from the most dominant IMF, $c_{2}$ (Fig. 3 (c)). The instantaneous frequency is superimposed on the WT plot in Fig. 3 (d), depicting that the lift coefficient in $t \in[0.25,1.25]$ is dictated by the unsteady aerodynamic excitation.

\section{B. Aeroelastic responses with structural damping}

When there exists a structural damping, the aeroelastic responses exhibit LCOs (i.e., steady-state, periodic motions), which is evident in Figs. 4 (a) and (b). The harmonics developed become much simpler than that in the case of no damping (Fig. 4 (c)) - only at odd factors of $\Omega=3 \omega_{1}$. From the WTs in Fig. 4 (c), we can see that the LCOs are formed after very short transients. The three frequency components for both modes are depicted in Figs. 4 (d) and (e), by means of the enhanced EMD analysis. The original responses are reconstructed in Fig. 4 (f), using all the three IMFs. However, the first IMF $\left(\approx \mathrm{e}^{5 j \Omega t}\right)$ can be negligible for the whole dynamics, and the next two components can be considered to be dominant.

The partial time series of the total lift coefficient in the presence of structural damping is plotted in Fig. 5 (a). Similar arguments to the case of no damping can be addressed, except for the fact that the presence of damping seems to eliminate the initial burst peak due to the aerodynamically dominated response. Instead, there appears continual smaller-amplitude, unsteady oscillatory response.

\section{Conclusions}

We have shown that aeroelastic response (e.g., heave and pitch displacements or total lift coefficient) represented as time histories can be decomposed onto a basis consisting of a small number of intrinsic mode functions obtained by standard techniques of empirical mode decomposition. These IMFs show promise for use in constructing reduced-order models (ROMs) of such systems, capable of capturing important interac-

tions, for example energy flow between substructures ${ }^{10,11,12}$, with a minimum of degrees of freedom. The properties of these ROMs, and their use in design, will be investigated in subsequent work.

\section{Acknowledgments}

This work was funded in part by U.S. Air Force Office of Scientific Research Contract Number FA9550 04-1-0073. DMM's participation was further supported by the Summer Faculty Program of the Air Force Research Laboratory. GK is supported by a grant from the Belgian National Science Foundation. All of these sources are gratefully acknowledged.

\section{References}

[1] Kerschen, G., Vakakis, A. F., Lee, Y. S., McFarland, D. M., and Bergman, L. A., "Toward a Fundamental Understanding of the Hilbert-Huang Transform," International Modal Analysis Conference XXIV, St. Louis, Missouri, 30 Jan.- 2 Feb. 2006.

[2] Kerschen, G., Vakakis, A. F., Lee, Y. S., McFarland, D. M., and Bergman, L. A., "Nonlinear MDOF System Characterization and Identification using the Hilbert-Huang Transform: Experimental Demonstration," International Modal Analysis Conference XXV, Orlando, Florida, 19-22 Feb. 2007. 

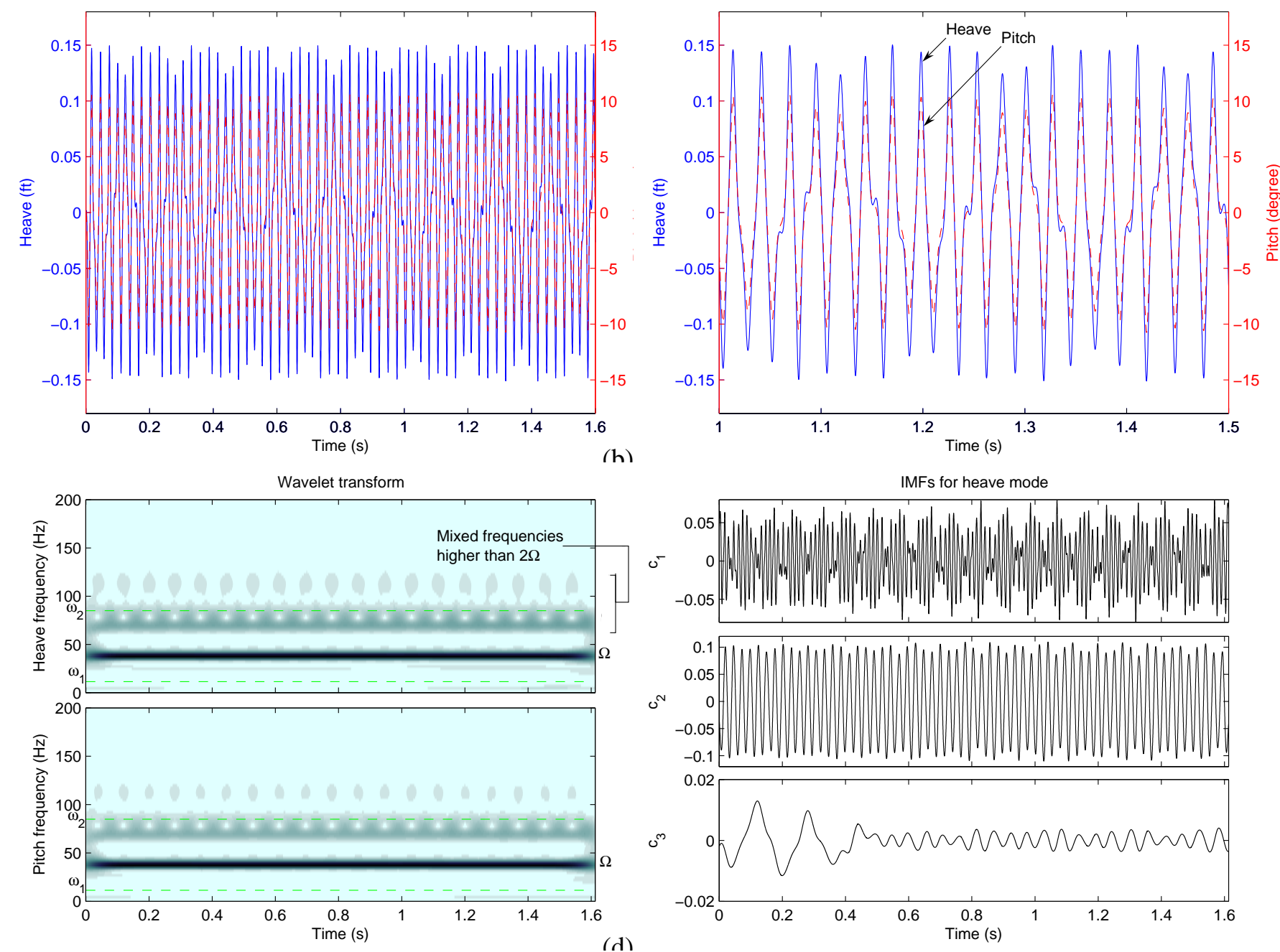

(c)

IMFs for pitch mode
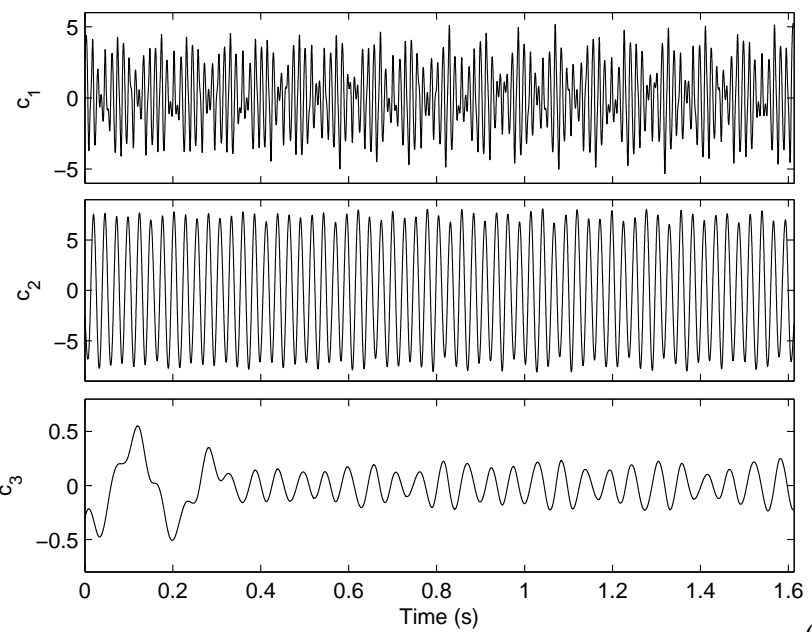

(e)
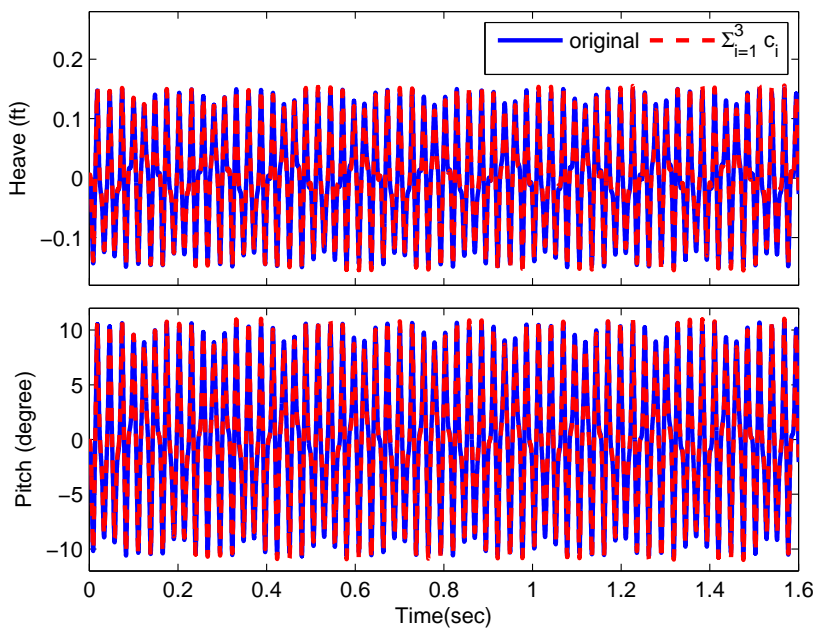

Fig. 2. Quasiperiodic flutter response when there is no structural damping present: (a) and (b) Comparison of the heave and pitch responses; (c) wavelet transforms; (d) and (e) the first three IMFs for the heave and pitch responses; (f) reconstruction of the original responses using the three IMFs. 

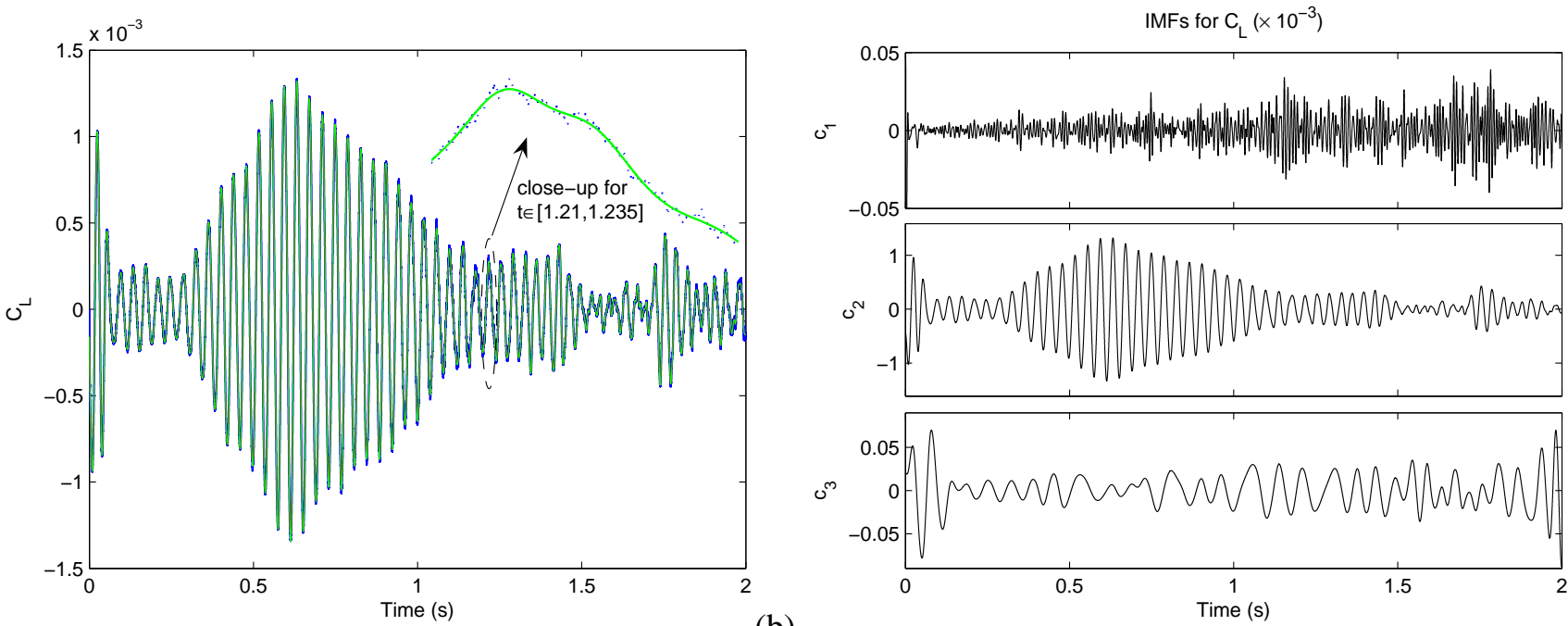

(a)

(h)

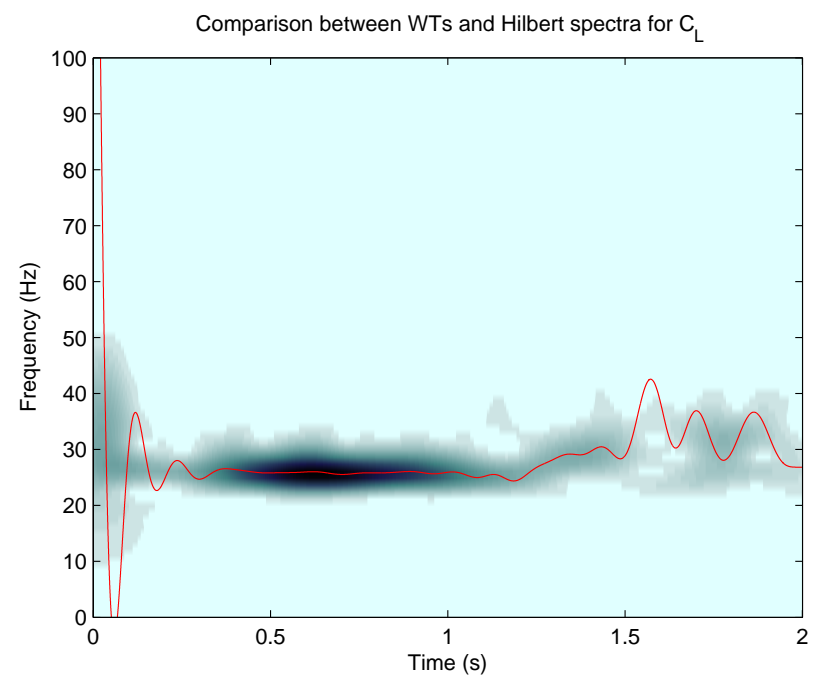

(c)

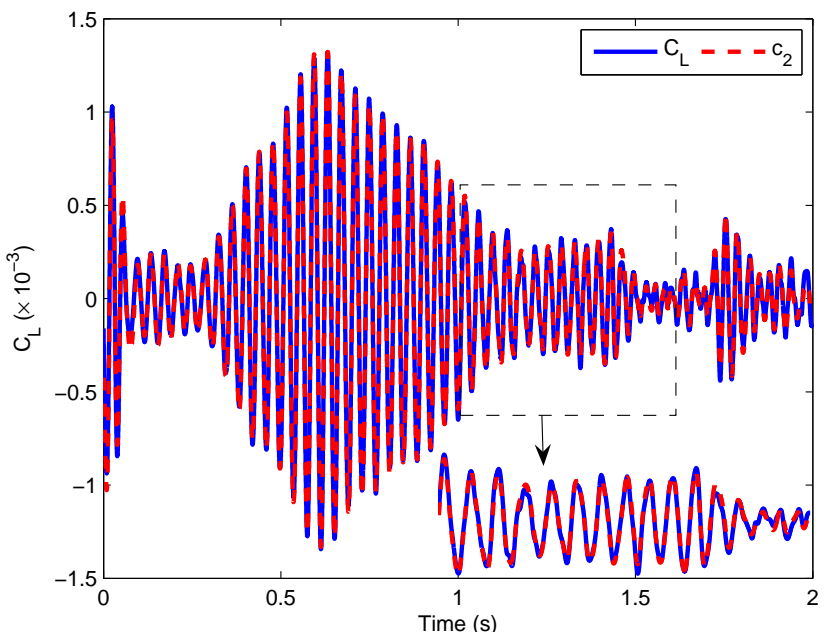

(d)

Fig. 3. Lift coefficient when there is no structural damping present: (a) Smoothening process for the response; (b) the three predominant IMFs; (c) reconstruction of the original response with the most dominant IMF; (d) instantaneous frequency of the dominant IMF superimposed on the WT. 

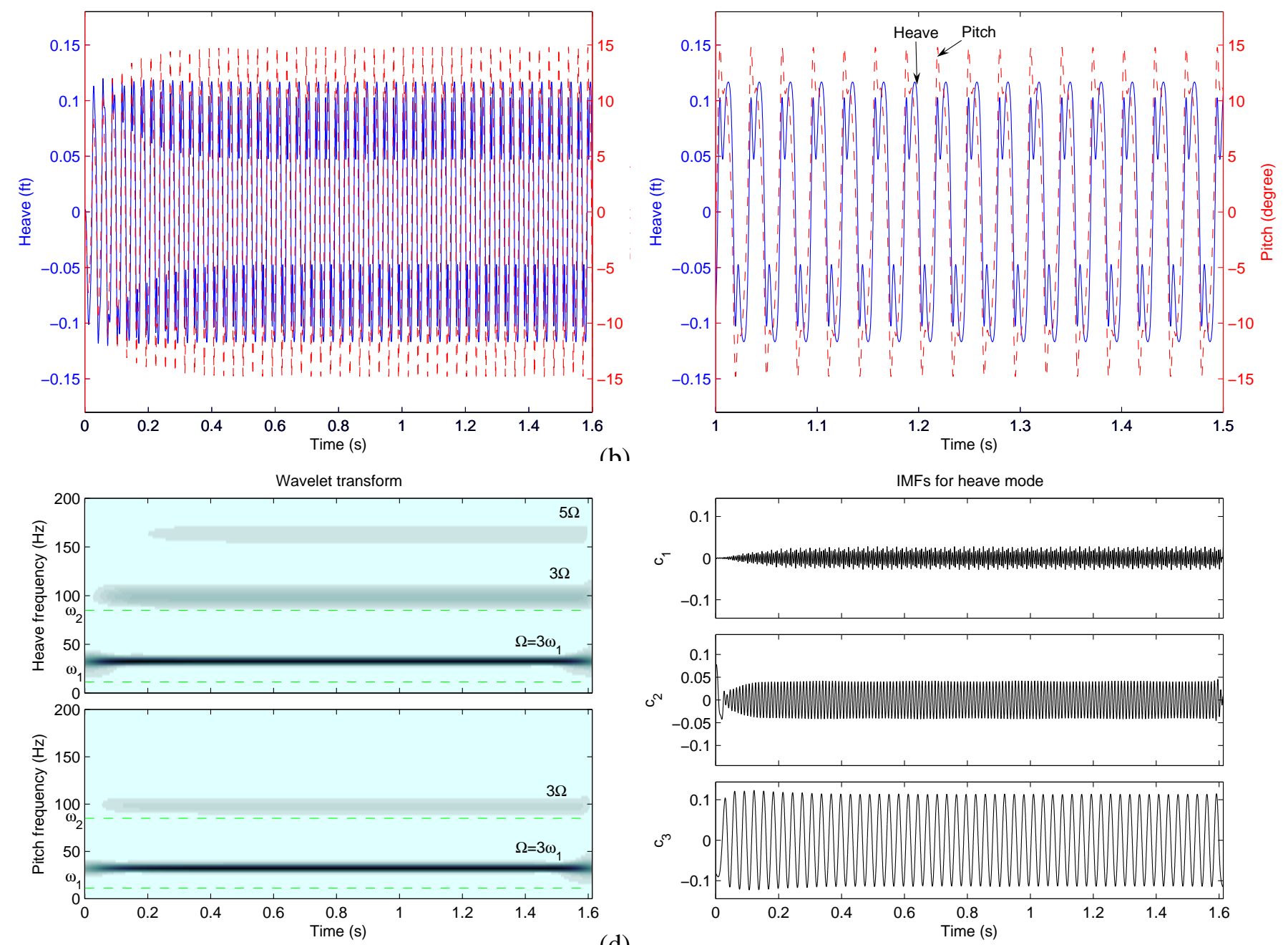

(c)
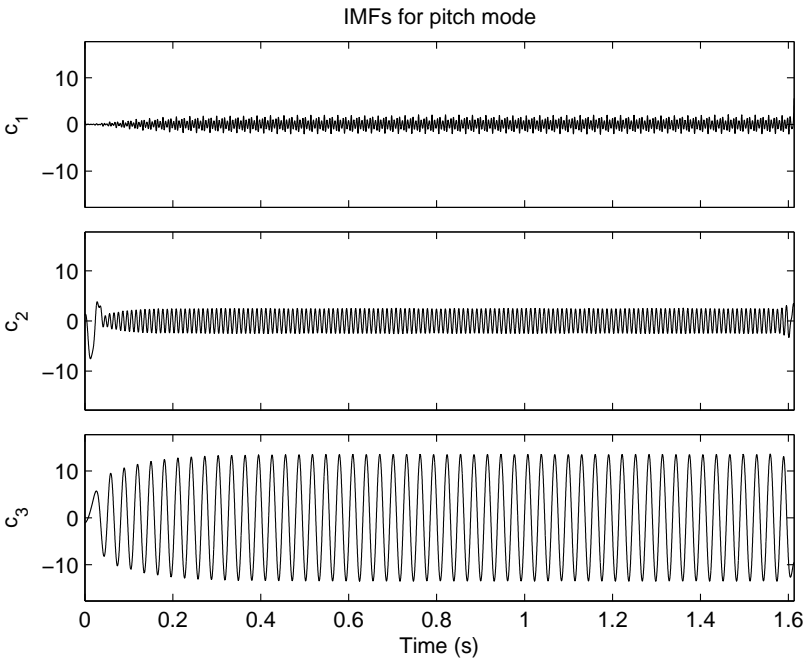

(d)
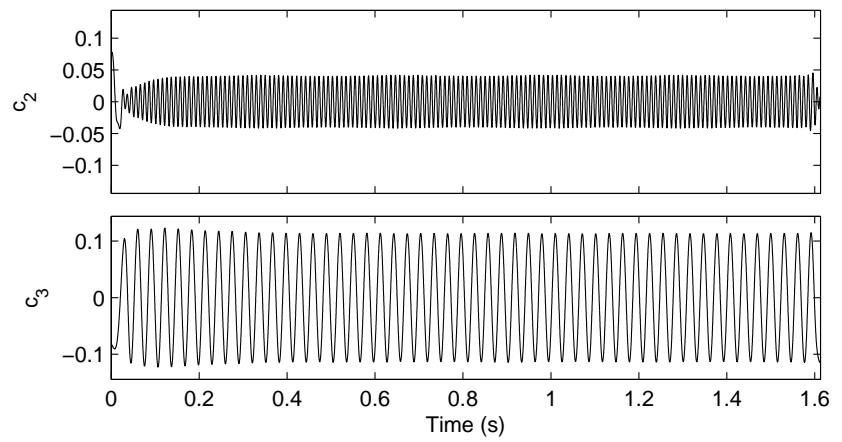

(e)

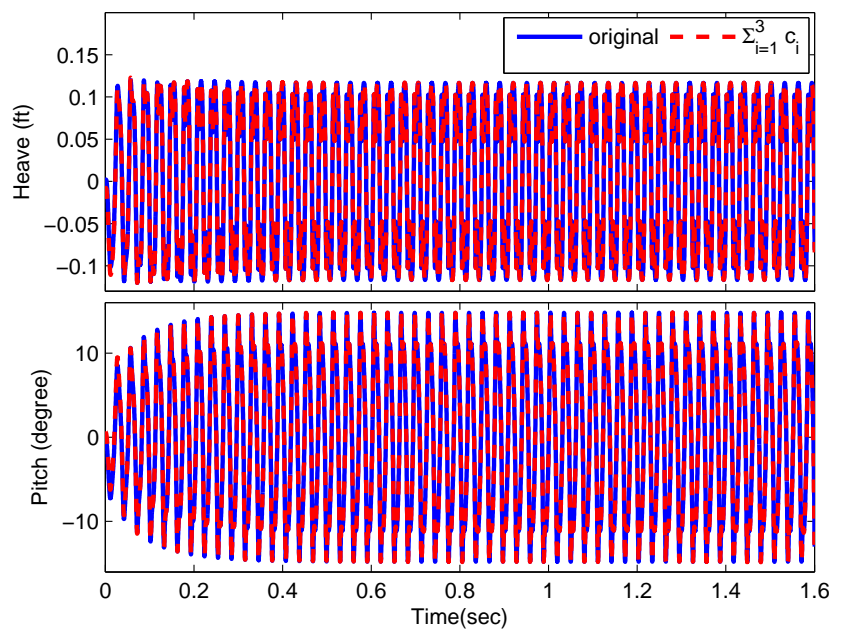

(f)

Fig. 4. LCO response when there is damping present: (a) and (b) Comparison of the heave and pitch responses; (c) wavelet transforms; (d) and (e) the first three IMFs for the heave and pitch responses; (f) reconstruction of the original responses using the three IMFs. 

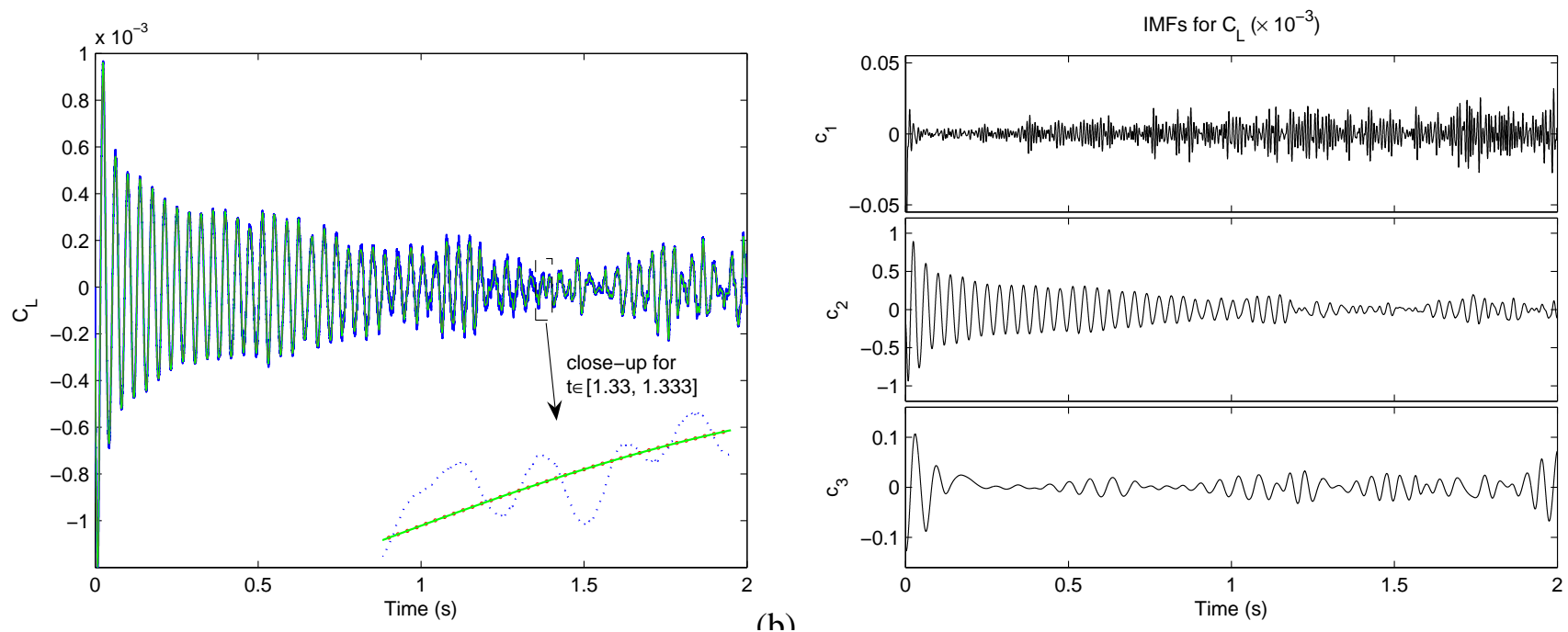

(a)

(h)

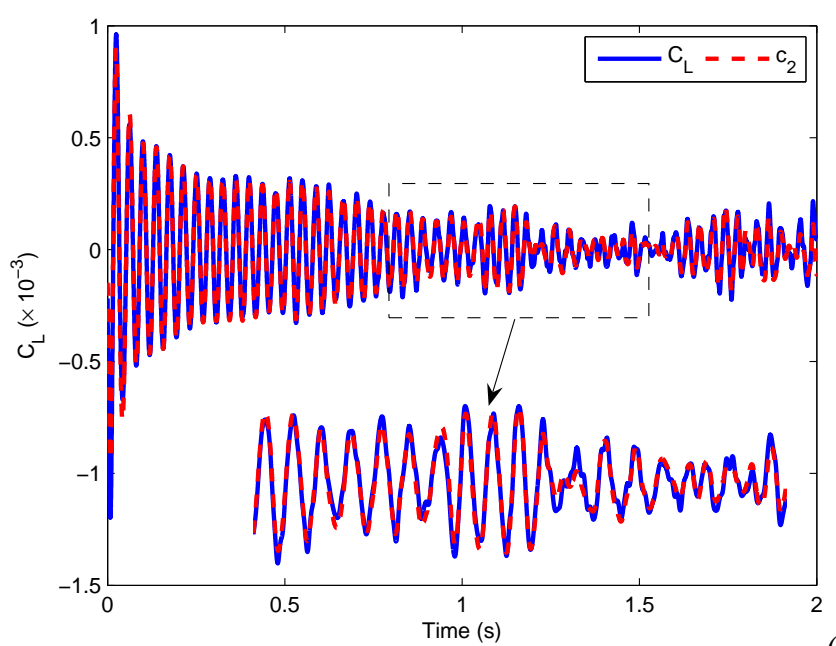

(c)

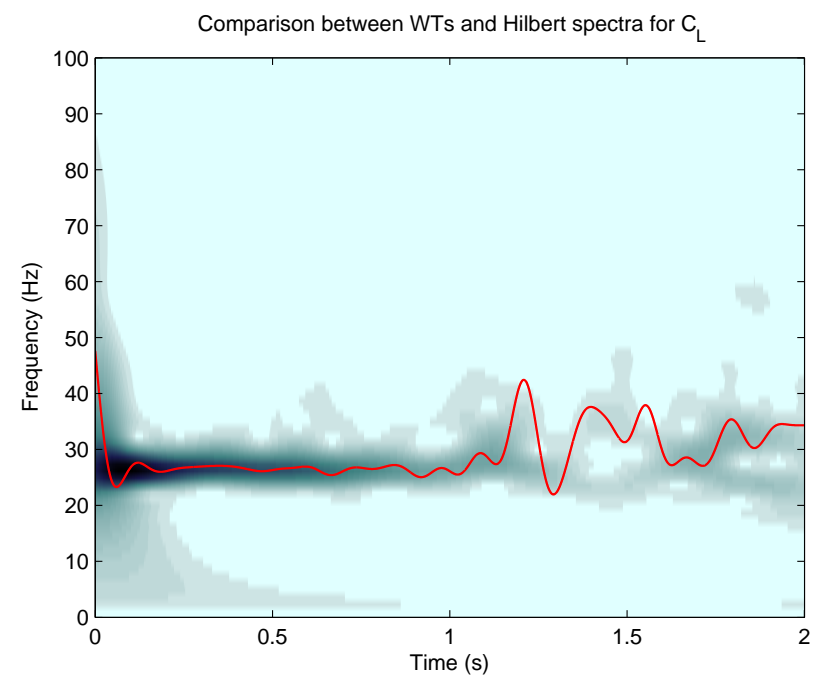

(d)

Fig. 5. Lift coefficient when there is damping present: (a) Smoothening process for the response; (b) the three predominant IMFs; (c) reconstruction of the original response with the most dominant IMF; (d) instantaneous frequency of the dominant IMF superimposed on the WT. 
[3] Kerschen, G., Vakakis, A. F., Lee, Y. S., McFarland, D. M., and Bergman, L. A., "The Slow-Flow Method of Identification in Nonlinear Structural Dynamics," 14th SPIE International Symposium on Smart Structures and Materials \& Nondestructive Evaluation and Health Monitoring, San Diego, California, 18-22 March 2007, Paper No. 652953.

[4] Manevitch, L. I., Mathematical Models of Nonlinear Excitations, Transfer Dynamics and Control in Condensed Systems, chap. Complex Representation of Dynamics of Coupled Oscillators, Kluwer Academic/Plenum Publishers, New York, 1999, pp. 269-300.

[5] Isogai, K., "On the Transonic-Dip Mechanism of Flutter of a Sweptback Wing," AIAA Journal, Vol. 17, No. 7, July 1979, pp. 793-795.

[6] Isogai, K., "Transonic Dip Mechanism of Flutter of a Sweptback Wing: Part II," AIAA Journal, Vol. 19, No. 9, Sept. 1981, pp. 1240-1242.

[7] Batina, J. T., "A Finite-Difference Approximate-Factorization Algorithm for Solution of the Unsteady Transonic Small-Disturbance Equation,” Tech. Rep. TP 3129, NASA, Jan. 1992.

[8] Beran, P. S., Khot, N. S., Eastep, F. E., Strganac, T. W., and Zweber, J. V., "Effects of Viscosity on Store-Induced Limit-Cycle Oscillation," ITEA Aircraft-Stores Compatibility Symposium, 18-21 Feb. 2003 .

[9] Khot, N. S., Beran, P. S., Zweber, J. V., and Eastep, F. E., "Influence of Tip Store Mass Location on Wing Limit-Cycle Oscillation (Paper AIAA 2003-1731)," Proceedings of the AIAA Dynamics Specialist Conference, April 2003.

[10] Lee, Y. S., Vakakis, A. F., Bergman, L. A., McFarland, D. M., and Kerschen, G., "Suppressing Aeroelastic Instability Using Broadband Passive Targeted Energy Transfers, Part I: Theory," AIAA Journal, Vol. 45, No. 3, March 2007, pp. 693-711.

[11] Lee, Y. S., Kerschen, G., McFarland, D. M., Hill, W. J., Nichkawde, C., Strganac, T. W., Bergman, L. A., and Vakakis, A. F., "Suppressing Aeroelastic Instability Using Broadband Passive Targeted Energy Transfers, Part II: Theory," AIAA Journal to appear, 2007.

[12] Lee, Y. S., Vakakis, A. F., Bergman, L. A., McFarland, D. M., and Kerschen, G., "Enhancing Robustness of Aeroelastic Instability Suppression Using Multi-Degree-of-Freedom Nonlinear Energy Sinks," AIAA Journal in review, 2007. 